\title{
Development and Validation of a Sonographic Enthesitis Instrument in Psoriatic Arthritis: The GRAPPA Diagnostic Ultrasound Enthesitis Tool (DUET) Project
}

\author{
Lihi Eder (i), Gurjit S. Kaeley (D), and Sibel Zehra Aydin (iD)
}

\begin{abstract}
Enthesitis is a key feature in psoriatic arthritis (PsA) and may be the initial site of musculoskeletal inflammation in patients with PsA. Ultrasound (US) optimizes the detection of enthesitis, but the lack of a validated sonographic enthesitis scoring system for PsA limits the ability to conduct US-based studies of approaches to improve the early diagnosis of PsA. Creating a sonographic enthesitis scoring system that reliably identifies PsA at early stages is an important step in optimizing early diagnosis and encouraging timely interventions that will ultimately improve longterm outcomes for patients with PsA. The Group for Research and Assessment of Psoriasis and PsA (GRAPPA) US working group has set a goal of improving the evaluation of enthesitis in patients with PsA by using US through the development of a Diagnostic Ultrasound Enthesitis Tool (DUET). This article summarizes the proposed DUET study design and methodology as discussed during the 2019 GRAPPA annual meeting in Paris, France. (J Rheumatol Suppl. 2020 June;96:50-2; doi:10.3899/jrheum.200128)
\end{abstract}

Key Indexing Terms:

PSORIASIS PSORIATIC ARTHRITIS GRAPPA ENTHESITIS ULTRASOUND

Delays in the diagnosis of psoriatic arthritis (PsA) remain a major gap in the care of the disease and contribute to the development of joint damage and poor longterm outcomes ${ }^{1}$. These delays are explained, in part, by the lack of reliable tools that could distinguish PsA from psoriasis alone and other rheumatic conditions. Enthesitis is a key feature in PsA and may be the initial site of musculoskeletal (MSK) inflammation ${ }^{2,3}$. Ultrasound (US) optimizes the detection of enthesitis, but the lack of a validated sonographic enthesitis scoring system that minimizes important confounders for PsA limits the ability to conduct US-based studies of approaches to improve the early diagnosis of $\mathrm{PsA}^{4}$. Creating a sonographic enthesitis scoring system that reliably identifies PsA at early stages would be an important step in (1) optimizing the early diagnosis of PsA, and (2) promoting

From the University of Toronto and Women's College Hospital, Toronto, Ontario, Canada; University of Florida College of Medicine, Jacksonville, Florida, USA; University of Ottawa, Ottawa Hospital Research Institute, Ottawa, Ontario, Canada.

As part of the supplement series GRAPPA 2019, this report was reviewed internally and approved by the Guest Editors for integrity, accuracy, and consistency with scientific and ethical standards.

L. Eder, MD, PhD, Assistant Professor of Medicine, University of Toronto and Women's College Hospital; G.S. Kaeley, MD, MBBS, MRCP, RhMSUS, Professor of Medicine, University of Florida College of Medicine; S.Z. Aydin, MD, Associate Professor of Medicine, University of Ottawa, Ottawa Hospital Research Institute.

Address correspondence to Dr. L. Eder, Women's College Hospital, 76 Grenville St., Toronto, Ontario M5S 1B2, Canada.

E-mail:Lihi.EDER@wchospital.ca timely interventions that would ultimately improve longterm PsA outcomes.

The Group for Research and Assessment of Psoriasis and PsA (GRAPPA) US working group includes GRAPPA members who have expertise in MSK US, methodology experts, and patient research partners from many countries. The working group has set a goal to optimize the evaluation of enthesitis in patients with PsA using US through the development of a Diagnostic Ultrasound Enthesitis Tool (DUET) ${ }^{5}$. During the 2019 GRAPPA annual meeting in Paris, France, the GRAPPA US working group met to discuss the general study design and the operational aspects of the project. This article describes the general design and methods of the DUET study as discussed in the meeting.

\section{Objectives of the DUET Study}

The global objective of the DUET study is to create a reliable sonographic enthesitis tool that distinguishes PsA from non-PsA patients, and to then assess its validity and reliability. The GRAPPA US working group will conduct the study as an international multicenter study. The new instrument will be developed through a combined data- and expert-driven approach that uses prospectively collected sonographic and clinical data. Ultimately, the working group anticipates that the new instrument will be (1) reliable, valid, and feasible for use in research as well as in the clinical care of individual patients; and (2) able to help improve the detection of PsA at early phases of the disease to ensure

Personal non-commercial use only. The Journal of Rheumatology Copyright @ 2020 . All rights reserved. 
that appropriate treatment can be administered in a timely fashion, thereby improving longterm disease outcomes.

The aim of the new sonographic enthesitis scoring instrument (DUET) is to distinguish between PsA and non-PsA patients. In contrast to existing sonographic scoring systems that are entirely expert-based ${ }^{6,7}$, the working group plans to use a combined iterative expert- and data-driven approach to develop the new scoring method. In a data-driven approach, a statistical process informs the development of the instrument and includes the optimal selection of items and item weights. This has the advantage of avoiding redundant items and improves the instrument's performance. However, to ensure the new instrument's face validity, expert opinion has been considered in the initial selection of variables, which reflects the "sonographic enthesitis" concept that will be evaluated in this study. In addition, expert opinion will guide the selection of the final scoring instrument from the top optimal combination of elementary sonographic lesions and entheseal sites to ensure face validity and feasibility.

\section{DUET Methodology}

The development of the new score will be based on prospectively collected data. The working group will collect sonographic and clinical information about the following 3 groups: (1) PsA cases, (2) psoriasis with no MSK symptoms, and (3) patients with noninflammatory rheumatic conditions without psoriasis. This information will be used to derive a sonographic tool that distinguishes PsA from other conditions. The diagnosis of PsA will be based upon the opinion of the rheumatologists prior to performing the US assessment, to avoid the influence of the US results on the rheumatologist's decision. Because the purpose of the new tool is to assist with the new diagnosis of PsA, the working group will restrict the population of PsA cases to patients with a recent rheumatologist diagnosis of PsA ( $<5$ yrs). Patients who are currently using biologic medications will be excluded, because these medications affect sonographic enthesitis.

Two control groups will be recruited: (1) patients with psoriasis without MSK symptoms, and (2) patients with noninflammatory rheumatic conditions without psoriasis. The choice of appropriate controls was carefully considered. Because the study's objective is to develop a method to distinguish PsA from other noninflammatory rheumatic conditions, particularly in the context of preexisting psoriasis, the 2 control groups are appropriate. These control groups represent conditions that are considered in the differential diagnosis of PsA and can be associated with entheseal abnormalities. The working group decided not to include patients with psoriasis and MSK symptoms as controls because they represent an intermediate group with "imminent PsA." Therefore, for the development of a diagnostic tool, it is more appropriate to include patients with well-defined, stable phenotypes.

\section{Acquisition and Scoring of US Scans}

Because of the high variability in US performance, much emphasis will be placed on harmonizing US acquisition and reading. Expert sonographers ( $>5$ yrs of experience in MSK US) who use high-quality US equipment have been invited to take part in this study. Training materials have been developed, including atlas and video clips that demonstrate standard scanning techniques for each entheseal site. The sonographers will be required to participate in a prestudy workshop aimed at ensuring standard US scanning techniques and image acquisition among all investigators.

The DUET study will evaluate 8 entheseal sites bilaterally (total 16 sites): quadriceps insertion at superior pole of patella, patellar ligament origins at the inferior pole of patella, patellar ligament insertion at the tibial tuberosity, Achilles tendon insertion into calcaneus, plantar fascia insertion into calcaneus, triceps tendon insertion into olecranon tuberosity, common extensor tendon insertion into lateral epicondyle, and supraspinatus insertion into superior facet of greater tubercle of humerus. The selection of entheseal sites was based on several considerations including knowledge from the working group's pilot study regarding informative entheseal sites in $\mathrm{PsA}^{8}$, existing clinical and sonographic entheseal scoring systems, and technical limitations of assessing specific entheseal sites. The guiding principle was to assess as many potentially informative entheseal sites as possible in the study to select the optimal combination of sites for a final score based on their discriminative ability.

The DUET study will use central and local reading methods to score entheseal lesions. The central reading will be the primary method used for analysis and development of the scoring tool. US files will be submitted for central reading, which will be performed independently by 2 of 3 readers. This method is expected to reduce interrater variability and to ensure blinding to the disease status. Local reading will also be performed by each local sonographer following a standard scoring protocol. Local scoring will be analyzed as secondary analysis for assessment of the validity and generalizability of the developed scoring tool.

\section{Planned Statistical Analysis}

The statistical analysis aims to obtain a limited set of variables, optimally chosen and weighted, with satisfactory discriminatory ability (the ability to discriminate between patients with PsA vs controls). The working group will fit a series of individual regression models for each of the US elementary lesions and entheseal sites evaluated in the study to identify those that contribute the most to discrimination between PsA and controls. This will allow the group to narrow the number of sonographic sites and possibly also the sonographic elementary lesions included in the final score, which will contribute to its feasibility. The regression coefficients will inform the appropriate weight of each element. The working group will (1) evaluate the area under the

Personal non-commercial use only. The Journal of Rheumatology Copyright @ 2020 . All rights reserved. 
receiver-operating curve for the model, (2) identify several cutoff points to discriminate between PsA and controls, and (3) calculate the tool's performance regarding sensitivity, specificity, and positive and negative predictive value. The working group will evaluate how known confounders, such as age groups, sex, and body mass index, affect the new tool's performance. To assess the construct validity of the new tool, the group will evaluate its correlation with clinical measures of disease activity (e.g., total enthesitis count and joint count) and patient-reported outcomes.

\section{Expected Effect of the DUET Study}

Optimizing the early detection of PsA among patients with psoriasis has important and direct implications for the advancement of clinical care and the improvement of healthcare outcomes for individuals with PsA. The DUET study is structured to provide a reliable tool for identifying patients with PsA in rheumatology and dermatology settings. The newly developed tool is expected to improve the early detection of PsA by helping the clinician to distinguish between PsA and other similar conditions. Early diagnosis may lead to early interventions, which will ultimately result in better control of disease activity and improved longterm disease outcomes. Recent advances in US technology, which have resulted in highly mobile, affordable devices, have the potential to significantly increase access to point-of-care US beyond the rheumatology setting. Therefore, having a reliable and feasible instrument is a critical first step before US-based studies can be conducted to assess the effect of new models of care and therapeutic interventions for early PsA.

\section{REFERENCES}

1. Haroon M, Gallagher P, FitzGerald O. Diagnostic delay of more than 6 months contributes to poor radiographic and functional outcome in psoriatic arthritis. Ann Rheum Dis 2015;74:1045-50.

2. McGonagle D, Gibbon W, Emery P. Classification of inflammatory arthritis by enthesitis. Lancet 1998;352:1137-40.

3. McGonagle DG, Helliwell P, Veale D. Enthesitis in psoriatic disease. Dermatology 2012;225:100-9.

4. Elalouf O, Bakirci Ureyen S, Touma Z, Anderson M, Kaeley GS, Aydin SZ, et al. Psoriatic arthritis sonographic enthesitis instruments: a systematic review of the literature. J Rheumatol 2019;46:43-56.

5. Eder L, Kaeley GS, Aydin SZ. The GRAPPA Sonographic Enthesitis Workshop. J Rheumatol Suppl. 2019 Jun;95:51-3.

6. Balint PV, Kane D, Wilson H, McInnes IB, Sturrock RD. Ultrasonography of entheseal insertions in the lower limb in spondyloarthropathy. Ann Rheum Dis 2002;61:905-10.

7. de Miguel E, Munoz-Fernandez S, Castillo C, Cobo-Ibanez T, Martin-Mola E. Diagnostic accuracy of enthesis ultrasound in the diagnosis of early spondyloarthritis. Ann Rheum Dis 2011; 70:434-9.

8. Tom S, Zhong Y, Cook R, Aydin SZ, Kaeley G, Eder L. Development of a preliminary ultrasonographic enthesitis score in psoriatic arthritis - GRAPPA Ultrasound Working Group. J Rheumatol 2019;46:384-90. 